\title{
Variational Homotopy Perturbation Method for Solving Riccati Type Differential Problems
}

\author{
Bothayna S. Kashkari, Sharefah Saleh \\ Department of Mathematics, Faculty of Sciences, King Abdulaziz University, Jeddah, Saudi Arabia \\ Email: bkashkari@kau.edu.sa, cabas@stu.kau.edu.sa
}

How to cite this paper: Kashkari, B.S. and Saleh, S. (2017) Variational Homotopy Perturbation Method for Solving Riccati Type Differential Problems. Applied Mathematics, 8, 893-900.

https://doi.org/10.4236/am.2017.87070

Received: May 26, 2017

Accepted: July 1, 2017

Published: July 4, 2017

Copyright () 2017 by authors and Scientific Research Publishing Inc. This work is licensed under the Creative Commons Attribution International License (CC BY 4.0).

http://creativecommons.org/licenses/by/4.0/

\begin{abstract}
In this paper, a Variational homotopy perturbation method is proposed to solve nonlinear Riccati differential equation. By combining the Variational Iteration Method and the Homotopy Perturbation Method, this technique possesses a fast convergence rate with high accuracy. The results reveal that the proposed method is very effective and simple.
\end{abstract}

\section{Keywords}

Riccati Equation, Homotopy Perturbation Method, Variational Iteration Method, Variational Homotopy Perturbation Method

\section{Introduction}

The Riccati equation plays a great role in blueprint and analysis the linear and nonlinear optimal control problems. Numerical Solution of this equation has been acquired by applying Adomian's decomposition method [1], homotopy Analysis method HAM [2], variational iteration method VIM [3] and homotopy perturbation method HPM [4]. HPM introduced by He [5], it can solve a large class of nonlinear problems activity, accurately and easily.

The application of HPM on nonlinear problems has been implemented by scientists and engineers, because this method is to continuously deform a difficult problem under study into a simple problem easy to solve. VIM proved by JiHuan He [5]. It is simple and powerful method for solving a broad type of nonlinear Problem. It was shown that this method is operative and reliable analytic and numerical purposes. The method gives rapidly convergent successive approximation of the exact solution if such solution existed.

The nonlinear Riccati differential equation [6] has following form

$$
\begin{aligned}
& L(x)=A(x) u^{2}(x)+B(x) u(x)+C(x), 0 \leq x \leq X \\
& u(0)=\alpha
\end{aligned}
$$


where $L=\frac{\mathrm{d}}{\mathrm{d} x}$ or $\frac{\mathrm{d}^{2}}{\mathrm{~d} x^{2}}, A(x), B(x)$ and $C(x)$ are continuous functions and $\alpha$ is an arbitrary constant.

We organize the following paper as follows. In Section 2, we present the VIM, while in Section 3, we present the HPM. In Section 4, we apply the VHPM to solve quadratic Riccati equation. Moreover, we find solutions of some examples by VHPM in Section 5.

The results reveal that the proposed method is very effective and simple. We end this paper by conclusion that reveal that these methods are very effective and convenient for solving nonlinear Riccati equations.

\section{Variational Iteration Method}

To illustrate the basic concepts of the VIM we consider the following differential equation [7] [8]

$$
L(u)+N(u)=f(x)
$$

where $L$ is a linear operator, $N$ is a nonlinear operator, and $f(x)$ is an inhomogeneous term. Then, we can construct the correct functional as follows

$$
u_{n+1}=u_{n}+\int_{0}^{x} \lambda(\xi)\left[L\left(u_{n}\right)+N\left(\tilde{u}_{n}\right)-f(\xi)\right] \mathrm{d} \xi
$$

where $\lambda$ is a general Lagrangian multiplier defined as [9]

$$
\lambda(x, t)=\frac{(-1)^{m}}{(m-1) !}(x-t)^{m-1}, m \geq 1
$$

And $\tilde{u}_{n}$ are restricted variation which means $\delta \tilde{u}_{n}=0$. Consequently, the solution $u=\lim _{n \rightarrow \infty} u_{n}$.

\section{Homotopy Perturbation Method}

To explain this method, we construct the following function [10]

$$
A(u)-f(x)=0, x \in \Omega
$$

With boundary condition

$$
B(u, \partial u / \partial n)=0, x \in \Gamma
$$

where $A$ is a general differential operator, $B$ is a boundary operator, $f(x)$ is a known analytical function. The operator $A$ can be decomposed into two operators $L$ and $N$, where $L$ is a linear operator and $N$ is a nonlinear operator.

By using the homotopy technique, we construct a homotopy $u(x, p): \Omega \times[0,1] \rightarrow \mathbb{R}$ which are satisfies

$$
H(u, p)=(1-p)\left[L(u)-L\left(u_{0}\right)\right]+p[L(u)+N(u)-f(x)]=0
$$

or

$$
H(u, p)=L(u)-L\left(u_{0}\right)+p\left[L\left(u_{0}\right)+N(u)-f(x)\right]=0
$$

where $p \in[0,1]$ is an embedding parameter. $u_{0}$ is an initial approximation of 
solution of equation.

$$
L(u)+N(u)-f(x)=0
$$

We have

$$
H(u, 0)=L(u)+L\left(u_{0}\right)=0, H(u, 1)=A(u)-f=0
$$

The solution can be written as a power series in $p$, $u(x)=\lim _{p \rightarrow 1} u=u_{0}+p u_{1}+p^{2} u_{2}+\cdots$.

\section{Variational Homotopy Perturbation Method}

In this section, we apply the VHPM to Riccati Equation (1), we start this method by applying HPM in Equation (8) on Equation (1), we get

$$
L u_{n}(x)-L u_{0}(x)+p\left[L u_{0}(x)-A(x) u_{n}^{2}-B(x) u_{n}-C(x)\right]=0
$$

Now we using correction functional in Equation (5) to get

$$
u_{n+1}=u_{n}+\int_{0}^{x} \lambda(\xi)\left[L u_{n}-L u_{0}+p\left(L u_{0}-A(\xi) u_{n}^{2}-B(\xi) u_{n}-C(\xi)\right)\right] \mathrm{d} \xi
$$

We can obtain

$$
u_{n+1}=u_{0}+p \int_{0}^{x} \lambda(\xi)\left[L u_{0}-A(\xi) u_{n}^{2}-B(\xi) u_{n}-C(\xi)\right] \mathrm{d} \xi
$$

Now we can rewrite Equation (13) in the form

$$
\sum_{n=0}^{\infty} p^{n} u_{n}=u_{0}+p \int_{0}^{x}\left[\lambda(\xi)\left(L u_{0}-N \sum_{n=0}^{\infty} p^{n} \tilde{u}_{n}-C(\xi)\right)\right] \mathrm{d} \xi
$$

As we see, the procedure is formulated by the coupling of VIM and HPM [11] [12] [13]. A comparison of like powers of $p$ give solutions of various orders.

\section{Numerical Examples}

\subsection{Example}

Consider the following classical Riccati differential equation

$$
u^{\prime}(x)=-u^{2}+2 u+1
$$

With initial condition $u(0)=0$.

For the above differential equation, the exact solution [14] is previously known to be

$$
u(x)=1+\sqrt{2} \tanh \left(\sqrt{2} x+\frac{1}{2} \log \left(\frac{\sqrt{2}-1}{\sqrt{2}+1}\right)\right)
$$

The Taylor expansion of $u(x)$ about $x=0$ gives

$$
u(x)=x+x^{2}+\frac{1}{3} x^{3}-\frac{1}{3} x^{4}-\frac{7}{15} x^{5}-\frac{7}{45} x^{6}+\frac{53}{315} x^{7}+\cdots
$$

Suppose that the initial approximation is $u_{0}=x$.

To solve Equation (15), by the VHPM we substitution it in Equation (14)

$$
\sum_{n=0}^{\infty} p^{n} u_{n}=u_{0}-p \int_{0}^{x}\left[-2 \sum_{n=0}^{\infty} p^{n} u_{n}+\left(\sum_{n=0}^{\infty} p^{n} u_{n}\right)^{2}\right] \mathrm{d} \xi
$$


Here $\lambda=-1$.

By comparing the coefficient of like powers of $p$, we have

$$
\begin{aligned}
& p^{(0)}: u_{0}=x \\
& p^{(1)}: u_{1}=-\int_{0}^{x}\left(-2 u_{0}+u_{0}^{2}\right) \mathrm{d} \xi=x^{2}-\frac{1}{3} x^{3} \\
& p^{(2)}: u_{2}=-\int_{0}^{x}\left(-2 u_{1}+2 u_{0} u_{1}\right) \mathrm{d} \xi=\frac{2}{3} x^{3}-\frac{2}{3} x^{4}+\frac{2}{15} x^{5} \\
& p^{(3)}: u_{3}=-\int_{0}^{x}\left(-2 u_{2}+2 u_{0} u_{2}+u_{1}^{2}\right) \mathrm{d} \xi=\frac{1}{3} x^{4}-\frac{11}{15} x^{5}+\frac{17}{45} x^{6}-\frac{17}{315} x^{7}
\end{aligned}
$$

The other components of the VHPM can be determined in similar way. Finally, the approximate solution of Equation (15) is $u=u_{0}+u_{1}+u_{2}+u_{3}+\cdots$. Which converge to the exact solution in Equation (16).

\subsection{Example}

Consider the following quadratic Riccati differential equation

$$
u^{\prime}(x)=-2 \mathrm{e}^{x} u^{2}+2 \mathrm{e}^{2 x} u+\mathrm{e}^{x}-\mathrm{e}^{3 x}
$$

With initial condition $u(0)=1$.

For the above differential equation, the exact solution [14] is previously known to be

$$
u(x)=\mathrm{e}^{x}
$$

The Taylor expansion of $u(x)$ about $x=0$ gives

$$
u(x)=1+x+\frac{1}{2} x^{2}+\frac{1}{6} x^{3}+\frac{1}{24} x^{4}+\frac{1}{120} x^{5}+\frac{1}{720} x^{6}+\frac{1}{5040} x^{7}+\cdots
$$

Suppose that the initial approximation is $u_{0}=x+1$.

To solve Equation (20), by the VHPM we substitution it in Equation (14), then we get

$$
\sum_{n=0}^{\infty} p^{n} u_{n}=u_{0}-p \int_{0}^{x}\left[1+\sum_{n=0}^{\infty} \frac{(2 \xi)^{n}}{n !}\left(\sum_{n=0}^{\infty} p^{n} u_{n}\right)-2 \sum_{n=0}^{\infty} \frac{(2 \xi)^{n}}{n !}\left(\sum_{n=0}^{\infty} p^{n} u_{n}\right)^{2}+\sum_{n=0}^{\infty} \frac{(3 \xi)^{n}}{n !}-\sum_{n=0}^{\infty} \frac{\xi^{n}}{n !}\right] \mathrm{d} \xi
$$

Here $\lambda=-1$.

By comparing the coefficient of like powers of $p$, we have

$$
\begin{aligned}
p^{(0)}: u_{0}= & x+1 \\
p^{(1)}: u_{1}= & \frac{1}{2} x^{2}+\frac{1}{6} x^{3}+\frac{1}{24} x^{4}-\frac{1}{24} x^{5}-\frac{49}{270} x^{6}-\frac{37}{270} x^{7}-\frac{1091}{40320} x^{8} \\
& +\frac{247}{45360} x^{9}-\frac{1}{50400} x^{10} \\
p^{(2)}: u_{2}= & \frac{1}{10} x^{5}+\frac{5}{36} x^{6}+\frac{13}{126} x^{7}+\frac{23}{480} x^{8}+\frac{19}{2592} x^{9}-\frac{3389}{302400} x^{10}-\cdots \\
p^{(3)}: u_{3}= & -\frac{1}{20} x^{5}-\frac{5}{72} x^{6}-\frac{13}{252} x^{7}-\frac{1}{120} x^{8}+\frac{59}{1728} x^{9}+\frac{32119}{604800} x^{10}+\cdots \\
: &
\end{aligned}
$$

The other components of the VHPM can be determined in similar way. Final- 
ly, the approximate solution of Equation (20) is $u=u_{0}+u_{1}+u_{2}+u_{3}+\cdots$. Which converge to the exact solution in Equation (21).

\subsection{Example}

Consider the Riccati Type Painleve's First Transcendent equation [15]

$$
u^{\prime \prime}(x)=6 u^{2}+\mu x, \quad \mu=1
$$

With initial conditions $u(0)=1, u^{\prime}(0)=0$.

The above differential equation without known exactly solutions and we suppose that the initial approximation is $u_{0}=1+x^{2}$.

To solve Equation (25) by the VHPM, substitution it in Equation (14), then we get

$$
\sum_{n=0}^{\infty} p^{n} u_{n}=u_{0}+p \int_{0}^{x} \lambda(\xi)\left[2-6\left(\sum_{n=0}^{\infty} p^{n} u_{n}\right)^{2}-\xi\right] \mathrm{d} \xi
$$

In this example $\lambda=(\xi-x)$.

By comparing the coefficient of like powers of $p$, we have

$$
\begin{aligned}
p^{(0)}: u_{0}= & 1+x^{2} \\
p^{(1)}: u_{1}= & 2 x^{2}+\frac{1}{6} x^{3}+x^{4}+\frac{1}{5} x^{6} \\
p^{(2)}: u_{2}= & 2 x^{4}+\frac{1}{10} x^{5}+\frac{6}{5} x^{6}+\frac{1}{21} x^{7}+\frac{9}{35} x^{8}+\frac{2}{75} x^{10} \\
p^{(3)}: u_{3}= & \frac{8}{5} x^{6}+\frac{13}{105} x^{7}+\frac{1877}{1680} x^{8}+\frac{11}{210} x^{9}+\frac{11}{35} x^{10}+\frac{17}{1925} x^{11} \\
& +\frac{254}{5775} x^{12}+\frac{x^{14}}{325}
\end{aligned}
$$

The other components of the VHPM can be determined in similar way. Finally, the approximate solution of Equation (25) is $u \approx u_{0}+u_{1}+u_{2}+u_{3}+\cdots$. In Table 1 we present the comparison between the approximate solution founded by VHPM with Truncated Taylor series(TTS) [16], and Rational approximation (RA) [17].

\subsection{Example}

Consider the Riccati Type Painleve's Second Transcendent equation [15]

$$
u^{\prime \prime}(x)=2 u^{3}+x u+\mu, \mu=1
$$

With initial conditions $u(0)=1, u^{\prime}(0)=0$.

The above differential equation without known exactly solutions and we suppose that the initial approximation is $u_{0}=1+x^{2}$.

To solve Equation (28) by the VHPM, substitution it in Equation (14), then we get

$$
\sum_{n=0}^{\infty} p^{n} u_{n}=u_{0}+p \int_{0}^{x} \lambda(\xi)\left[1-2\left(\sum_{n=0}^{\infty} p^{n} u_{n}\right)^{3}-\xi \sum_{n=0}^{\infty} p^{n} u_{n}\right] \mathrm{d} \xi
$$


Table 1. Comparison between the approximate solution $u=\sum_{i=0}^{20} u_{i} \quad$ with TTS and RA.

\begin{tabular}{cccc}
\hline$X$ & VHPM & TTS & RA \\
\hline 0.0 & 1.000000 & 1.0000 & 1.0000 \\
0.1 & 1.030471 & 1.030471 & 1.0305 \\
0.2 & 1.126366 & 1.126366 & 1.1264 \\
0.3 & 1.301454 & 1.301453 & 1.3015 \\
0.4 & 1.583055 & 1.583054 & 1.5831 \\
0.5 & 2.022763 & 2.022771 & 2.0228 \\
0.6 & 2.721246 & 2.721242 & 2.7212 \\
0.7 & 3.890893 & 3.890886 & 3.8909 \\
0.8 & 6.038351 & 6.038340 & 6.0383 \\
0.9 & 10.622497 & 10.622610 & 10.6223 \\
1.0 & 23.363804 & 23.393600 & 23.3860 \\
\hline
\end{tabular}

And we have $\lambda=(\xi-x)$.

By comparing the coefficient of like powers of $p$, we have

$$
\begin{aligned}
p^{(0)}: u_{0}= & 1+x^{2} \\
p^{(1)}: u_{1}= & \frac{1}{2} x^{2}+\frac{1}{6} x^{3}+\frac{1}{2} x^{4}+\frac{1}{20} x^{5}+\frac{1}{5} x^{6}+\frac{1}{28} x^{8} \\
p^{(2)}: u_{2}= & \frac{1}{4} x^{4}+\frac{3}{40} x^{5}+\frac{11}{36} x^{6}+\frac{1}{51} x^{7}+\frac{41}{224} x^{8}+\frac{1}{224} x^{9} \\
& +\frac{131}{2100} x^{10}+\frac{47}{15400} x^{11}+\frac{19}{1540} x^{12}+\frac{3}{2548} x^{14} \\
p^{(3)}: u_{3}= & \frac{1}{10} x^{6}+\frac{17}{420} x^{7}+\frac{383}{2240} x^{8}+\frac{703}{12960} x^{9}+\frac{6887}{50400} x^{10} \\
& +\frac{4013}{123200} x^{11}+\frac{1039}{15840} x^{12}+\frac{2759}{257400} x^{13}+\frac{117269}{5605600} x^{14} \\
& +\frac{151}{77000} x^{15}+\frac{74933}{16816800} x^{16}+\frac{28671}{190590400} x^{17}+\frac{5653}{9529520} x^{18} \\
& +\frac{15}{387296} x^{20}
\end{aligned}
$$

The other components of the VHPM can be determined in similar way. Finally, the approximate solution of Equation (28) is $u \approx u_{0}+u_{1}+u_{2}+u_{3}+\cdots$. In Table 2 we present the comparison between the approximate solution founded by VHPM with TTS [15], and RA [17].

\section{Conclusion}

In this paper, we studied the solution of nonlinear Riccati differential equation. We have applied a recently introduced technique called the VHPM to solve this nonlinear differential equation. This method is more efficient and simpler. The results of the method exhibit excellent agreement with the exact solution. The 
Table 2. Comparison between the approximate solution $u=\sum_{i=0}^{20} u_{i} \quad$ with TTS and RA.

\begin{tabular}{cccc}
\hline$x$ & VHPM & TTS & RA \\
\hline 0.0 & 1.000000 & 1.0000 & 1.0000 \\
0.1 & 1.015244 & 1.01520 & 1.0152 \\
0.2 & 1.062614 & 1.06260 & 1.0626 \\
0.3 & 1.146376 & 1.14640 & 1.1464 \\
0.4 & 1.274152 & 1.27420 & 1.2742 \\
0.5 & 1.459213 & 1.45920 & 1.4592 \\
0.6 & 1.725376 & 1.72540 & 1.7254 \\
0.7 & 2.118444 & 2.11840 & 2.1184 \\
0.8 & 2.736936 & 2.73690 & 2.7369 \\
0.9 & 3.834399 & 3.83440 & 3.8343 \\
1.0 & 6.309968 & 6.31100 & 6.3104 \\
\hline
\end{tabular}

comparison between the numerical results with TTS and RA in Problems 3 and 4 of validates the accuracy of the VHPM method for problems without known exactly solutions that have been advanced for solving Riccati equation shows that the new technique is reliable and powerful.

\section{References}

[1] Gbadamosi, B., Adebimpe, O., Akinola, E.I. and Olopade, I.A. (2012) Solving Riccati Equation Using Adomian Decomposition Method. International Journal of Pure and Applied Mathematics, 78, 409-417.

[2] Tan, Y. and Abbasbandy, S. (2013) Homotopy Analysis Method for Quadratic Riccati Differential Equation. Communications in Nonlinear Science and Numerical Simulation, 1, 539-546.

[3] Ghorbani, A. and Momanib, Sh. (2010) An Effective Variational Iteration Algorithm for Solving Riccati Differential Equations. Applied Mathematics Letters, 23, 922-927. https://doi.org/10.1016/j.aml.2010.04.012

[4] Vahidi, A.R., Azimzadehand, Z. and Didgar, M. (2013) An Efficient Method for Solving Riccati Equation Using Homotopy Perturbation Method. Indian Journal of Physics, 87, 447-454. https://doi.org/10.1007/s12648-012-0234-8

[5] He, J.H. (2000) Variational Iteration Method for Autonomous Ordinary Differential Systems. Applied Mathematics and Computation, 114, 115-123.

https://doi.org/10.1016/S0096-3003(99)00104-6

[6] Reid, W.T. (1972) Riccati Differential Equations. Academic Press, New York.

[7] He, J.H. (2007) Variational Iteration Method: New Development and Applications. Computers and Mathematics with Applications, 54, 881-894.

https://doi.org/10.1016/j.camwa.2006.12.083

[8] He, J.H. (1999) Variational Iteration Method-A Kind of Non-Linear Analytical Technique: Some Examples. International Journal of Non-Linear Mechanics, 34, 699-708. https://doi.org/10.1016/S0020-7462(98)00048-1

[9] Fadhil, H., Saad, A., Bewar, A. and Yousif, A. (2015) Variationalhomotopy Perturbation Method for Solving Benjamin-Bena-Mahony Equation. Applied Mathemat- 
ics, 6, 675-683. https://doi.org/10.4236/am.2015.64062

[10] He, J.H. (1999) Homotopy Perturbation Method. Computer Methods in Applied Mechanics and Engineering, 178, 257-262. https://doi.org/10.1016/S0045-7825(99)00018-3

[11] Daga, A. and Pradhan, V. (2014) Variational Homotopy Perturbation Method for the Nonlinear Generalized Regularized Long Wave Equation. American. Journal of Applied Mathematics and Statistics, 2, 231-234.

[12] Abolarin, O.E. (2013) New Improved Variational Homotopy Perturbation Method for Bratu-Type problems. American Journal of Computational Mathematics, 3, 110-113. https://doi.org/10.4236/ajcm.2013.32018

[13] Easif, F.H., Manaa, S.A., Mahmood, B.A. and Yousif, A.M. (2015) Variational Homotopy Perturbation Method for Solving Benjamin-Bona-Mahony Equation. Applied Mathematics, 6, 675-683. https://doi.org/10.4236/am.2015.64062

[14] Aminikhah, H. (2013) Approximate Analytical Solution for Quadratic Riccati Differential Equation. Iranian Journal of Numerical Analysis and Optimization, 3, 21 31.

[15] Rasedee, A., Abdul Sathar, M., Deraman, F., Ijam, H., Suleiman, M., Saaludin, N. and Rakhimov A. (2016) 2 Point Block Backward Difference Method for Solving Riccati Type Differential Problems. AIP Conference Proceedings, 1775, Article ID: 030005. https://doi.org/10.1063/1.4965125

[16] Simon, W.E. (1965) Numerical Technique for Solution and Error Estimate for the Initial Value Problem. Mathematics of Computation, 18, 387-393. https://doi.org/10.1090/S0025-5718-1965-0179951-9

[17] Fair, W. and Luke, Y.L. (1966) Rational Approximations to the Solution of the Second Order Riccati Equation. Mathematics of Computation, 20, 602-606. https://doi.org/10.1090/S0025-5718-1966-0203906-X

\section{Submit or recommend next manuscript to SCIRP and we will provide best service for you:}

Accepting pre-submission inquiries through Email, Facebook, LinkedIn, Twitter, etc. A wide selection of journals (inclusive of 9 subjects, more than 200 journals)

Providing 24-hour high-quality service

User-friendly online submission system

Fair and swift peer-review system

Efficient typesetting and proofreading procedure

Display of the result of downloads and visits, as well as the number of cited articles

Maximum dissemination of your research work

Submit your manuscript at: http://papersubmission.scirp.org/

Or contact am@scirp.org 